\title{
WHAT IS PSYCHOPHYSIOLOGY? WHERE TO GO NEXT?
}

\author{
Kaneez Fatima Shad
}

Medical and Molecular Biosciences -University of Technology Sydney, Australia. Corresponding Author Email: Kaneez.Fatima-Shad@uts.edu.au

Psychophysiology is an area of science which helps us to differentiate between self-reported emotion and physiological expression of emotion. Many techniques such as EMG, EEG and MEG (Magnetoencephalograph) can be used for measuring muscular and electrical activities as well as event related potentials (ERP). EMG of facial muscles (e.g. corrugator, zygomatic, and levator labii superioris/alesque) patterning plus autonomic physiology (e.g. heart rate and skin conductance level) gives a basic picture of a person sense for certain situations. For example activity at a facial muscle, the levator labii was higher during disgust than during anger (Kuhl, 2008). Psychophysiology deals with the exploitation of psychological variables and their equivalent pragmatic effects on physiological processes. Thus, this discipline examines the interactions between physiological and psychological events. In general, psychophysiology studies the behavioral consequences of physiological properties of the body at a biochemical and anatomical level, and vice versa. In short this discipline examines the perception of emotion, behavioral states, stress, cognitive task performance, personality and intelligence. The initiation, execution, maintenance and termination of behavioral events can be determined by observing the relationships between psychological factors, stimulus perception and recognition, physiological response are used to better understand these perceptions. This discipline is generally divided into six major areas:

Developmental psychophysiology: deals with the changes in the physiological system with the age and related behavior. Usually brain activities such as event-related potentials (ERPs) are used to examine these behavioral changes.

Cognitive psychophysiology: This branch deals with the association between the cognitive task performance and physiological events. For example, it looks at how perception, movement, attention, language and memory may be associated with particular features of the brain electrical and magnetic activities. The vision of having an electrophysiological index of cognitive process has led to the exploration of P300 event of ERP and at present P300 amplitude and latency can be used as a guide for the nature and timing of a subject's cognitive response to a stimulus. Many reasons such as attentiveness, orientation, decision making have been suggested to explain the observed variation in P300. Ray Johnson described beautifully the three dimension relationship of event perception and P300 amplitude, indicating the importance of information transmission, subjective probability and stimulus meaning (Johnson, 1986).

Clinical psychophysiology: The study of psychological disorders and their relationship with physiology and pathophysiology. This area also deals with the observation for the effectiveness of treatment regimens and drug effects on the psychological behavior. Benefits of any treatment applied should be measured in terms of behavioral outcomes as well as changes observed both in physiological parameters and biochemical assays.

Page $\mid 1$

(CAdvance Education Institute \& Research Centre-2014
Applied psychophysiology: It is a branch which deals with the application of psychophysiological techniques to occupational, recreational and clinical areas. Applied Psychophysiologist monitors certain physiological activities within an individual, and provides instant and appropriate feedback called the biofeedback. This branch deals with a variety of practical problems such as stuttering, respiration control and can be cured by self-control therapeutic techniques.

Individual differences: This area looks specifically at the relation of physiological processes and anatomical structures to measure the personality and intelligence. In the past histological surveys of human cadavers' shows that the number of dendrites and their length are positively correlated with the level of education within individuals. At present we can measure such relationships by using different types of MRIs and other electrical activity measuring techniques.

Social psychophysiology: It is deals with the relationship between physiology and social behavior. For example an individual's group dynamics can be observed by monitoring the physiological changes such as pupil size, muscle tone and skin electrical resistance. All areas of psychophysiology require a number of measurement processes for describing the activity of a particular physiological system and its psychological outcome. In the muscular system, skeletal muscle activity is carried out by electromyography, in which the electrical potentials that are associated with contractions of muscle fibers are measured and can be assessed for their psychological effects. For example electromyographic activity is associated with real smiles when conducting easier mental tasks.

As mentioned in the beginning that autonomic physiology closely reflects the psychological effects and skin conductance and heart rate are good examples of such expressions. Electrodermal activity or galvanic skin response is used to measures the sweat gland action by evaluating the electrical properties of the skin that are associated with the gland. This skin response is receptive to any changes in emotional and cognitive activities. Likewise, pupillary response depicts the dilation of the pupil of the eye, while electrooculography describes the measurement of eye movement. In addition, eye blink rate and duration can be measured. Pupillary dilation is considered to be an indicative of subject's extreme interest and excitement, while electro-oculograms are regularly used in sleep research, for instance, as one indicator of entry to the phase of sleep known as REM (rapid eye movement) sleep. Electrocardiography refers to the recording of the electrical potentials generated by the heart muscles over the period of one heartbeat. The electrical waveform produced by the sequence of contractile responses in a heartbeat is referred to as the PQRST complex. Measures such as heart beat variability have been widely used to indicate the mental workload. Correspondingly, measures of the breathing and gas-exchange process are made by using oximetry for examining the arterial blood oxygen $(\mathrm{O} 2)$ levels and infrared capnometry for the lung carbon dioxide (CO2) levels. There is a strong relationship between respiration and 
psychological factors. An interesting finding from the analysis of respiration is that the individuals suffering from panic disorder have greater irregularity and complexity in their breathing patterns, which may make them more vulnerable to panic attacks. Similarly, the electrical activity generated by the neurons from the different areas of the brain can be measured by using electroencephalography and electrical currents generated magnetic fields, can be assessed by magnetoencephalography. Psychophysiological methods offer insight into a wide range of human behaviors and experiences with a range of techniques available to the psychophysiological researchers.
Now the questions are:

Where we are going to go from here?

Is this a journey from mind to molecules?

Or is there any organ other than brain in our body which is capable of dictating the behavioral features and cognitive outcomes?

REFERENCES:

- $\quad$ Kuhl, P., \& Rivera-Gaxiola, M. (2008). Neural substrates of language acquisition. Annu. Rev. Neurosci., 31, 511-534.

- Johnson, R. (1986). A triarchic model of P300 amplitude. Psychophysiology,

23(4), 367-384. 